\title{
A comparison of sublingual with vaginal administration of misoprostol for induction of labor at term
}

\author{
Monika D. Akare*, Purvi K. Patel
}

Department of Obstetrics and Gynecology, Medical College and Shree Sayaji General Hospital, Baroda, Gujarat, India

Received: 17 January 2017

Accepted: 27 February 2017

\author{
*Correspondence: \\ Dr. Monika D. Akare, \\ E-mail: dr.monicaakare@gmail.com
}

Copyright: (c) the author(s), publisher and licensee Medip Academy. This is an open-access article distributed under the terms of the Creative Commons Attribution Non-Commercial License, which permits unrestricted non-commercial use, distribution, and reproduction in any medium, provided the original work is properly cited.

\section{ABSTRACT}

Background: To compare the efficacy and safety of sublingual route of misoprostol with vaginal route of administration.

Methods: This study was conducted at Department of Obstetrics and Gynecology, Medical College, Baroda, Gujarat, India. 50 cases each with a singleton term pregnancy and a live fetus requiring induction of labor were allocated to sublingual and vaginal administration of misoprostol. Outcome measures related to labor and maternal and fetal side effects were compared between the 2 groups and evaluated using Chi square test and relative risk (RR) with 95\% confidence intervals $(\mathrm{CI})$.

Results: The sublingual route of misoprostol was associated with a reduced risk of failed induction, reduced time from initiation to induction, reduced induction to delivery interval and a higher incidence of maternal and fetal side effects. However, the differences were not statistically significant.

Conclusions: The sublingual route of administration of misoprostol is comparable in efficacy and safety to the vaginal route for induction.

Keywords: Induction of labour, Misoprostol, Sublingual

\section{INTRODUCTION}

Amongst the plethora of techniques available for induction of labor, Prostaglandins remain the single most effective means of achieving cervical ripening and inducing labor and have been administered through various routes. Pharmacological studies suggest that sublingual route might be the optimal route of administration for $\mathrm{PGE}_{1}$ analogue misoprostol because the avoidance of the first pass hepatic circulation would yield bioavailability similar to that achieved with the vaginal route along with an earlier onset of action and a prolonged activity. ${ }^{1-4}$ This has generated an interest in the sublingual route for labor induction. An additional possible advantage is that avoidance of direct cervical effects might reduce the risk of uterine hyperstimulation. Sublingual dosing for labor induction is attractive also because of ease of administration, less frequent need for vaginal examination, greater freedom of position and the possibility of its convenient use despite vaginal bleeding or ruptured membranes.

A few recent studies have found that sublingual administration of misoprostol is also effective for induction of labour. ${ }^{5-7}$ This study was designed to compare the efficacy and safety of tablet misoprostol 25 $\mu \mathrm{g}$ administered sublingually with that of routinely employed tablet misoprostol $25 \mu \mathrm{g}$ administered vaginally for induction of labor at term.

\section{METHODS}

This study was conducted at Department of Obstetrics and Gynecology, Medical College and Shree Sayaji 
General Hospital, Baroda, Gujarat, India over a one-year period. The study included 100 subjects. There were 50 cases each in both the groups i.e. sublingual and vaginal.

\section{Inclusion criteria}

- $\quad$ Full term pregnancy (>37 weeks gestation)

- Live fetus

- Singleton pregnancy

- Cephalic presentation

- Unfavorable cervix (Bishop's score $<6$ )

- Reassuring fetal heart tracing

- Absence of uterine contractions

\section{Exclusion criteria}

- Preterm pregnancy

- Intrauterine fetal death

- Multiple gestation

- Scarred uterus (previous caesarean section, myomectomy, hysterotomy)

- Non-reassuring fetal heart rate

- Grand Multi-parity (parity >4)

- Chorioamnionitis

\section{Indications for induction}

- Pre- eclampsia

- Premature rupture of membranes

- Oligohydramnios

- Postdatism

A detailed history, general physical examination and Obstetrical examination were carried out. Per vaginum examination was done for assessing bishop's score and pelvis. Subjects meeting the above criteria with no contraindication to vaginal delivery were allocated alternately in each group.

A written and informed consent was taken. In sublingual group, tablet misoprostol 25 micrograms $(\mu \mathrm{g})$ was held below the tongue till it got dissolved and in vaginal group, tablet misoprostol $25 \mu \mathrm{g}$ was kept in the posterior fornix of vagina. In both the groups, tablet misoprostol 25 $\mu \mathrm{g}$ was repeated every 4 hourly to a maximum of 5 doses (if required). Failure of induction was defined as unfavourable cervix after 5 doses (evaluated 4 hours after last dose). The subsequent dose was withheld in the presence of any of the following: at least three regular uterine contractions in 10 minutes, active phase of labor (defined as regular uterine contractions with cervical dilation $>3 \mathrm{~cm}$ ), cervix favorable for amniotomy (Bishop score $>8)$.

As soon as fetal head engagement and cervical dilation permitted, amniotomy was performed, followed by oxytocin augmentation if the frequency of contractions was less than three per 10 minutes or the contractions pattern was dysfunctional. Oxytocin was administered not earlier than 4 hours after the last misoprostol dose, starting at $1 \mathrm{mU} /$ minute and increased by $1 \mathrm{mU} / \mathrm{minute}$ every 15 minute until adequate contractions persisted. If the woman went into labor or if the Bishop's score was 8 or more, an artificial rupture of membranes was performed. Induction of labor was considered to have failed when cervix was unfavorable (Bishop's score <8) after 5 doses of misoprostol (evaluated 4 hours after last dose). If there was failure of induction, caesarean section was performed.

During entire intrapartum period strict monitoring of fetal heart rate and rhythm was done \& uterine activity was monitored for tachysystole, hypertonus and hyper stimulation syndrome. For the study, the term 'uterine hyperstimulation without FHR changes' included uterine tachysystole (greater than five contractions per 10 minutes for at least 20 minutes) and uterine hypersystole/ hypertonus (a contraction lasting at least two minutes) and 'uterine hyperstimulation with FHR changes' denoted uterine hyperstimulation syndrome (tachysystole or hypersystole with fetal heart rate changes such as persistent decelerations, tachycardia or decreased shortterm variability). All the episodes of hyperstimulation syndrome were included in the analysis regardless of the interval from the time of misoprostol administration to the occurrence of the abnormal FHR pattern. Recognized episodes of hyperstimulation and abnormal FHR pattern were managed by intrauterine resuscitation, which included stopping the oxytocin infusion, maternal repositioning, hydration and oxygen administration. When resuscitation failed to convert the fetal status to a reassuring one, emergency cesarean section for fetal distress was performed.

The primary outcome measure was the interval from start of induction to active phase. Secondary outcomes studied were need for oxytocin augmentation, interval from induction to delivery, vaginal delivery within 24 hours, total dose required for induction, mode of delivery, need for cesarean section with indication, uterine hyperstimulation with fetal heart rate (FHR) changes, meconium stained liquor, APGAR score less than seven at one minutes, maternal side effects and complications.

\section{Statistical analysis}

The data was analyzed with the help of computer software SPSS version 12.0 for windows. Statistically significant differences were evaluated using Chi square test. $\mathrm{P}$ value of $<0.05$ was considered as statistically significant. For discrete data, relative risk (RR) with $95 \%$ confidence intervals (CI) was used.

\section{RESULTS}

Both the groups were comparable with regards to maternal age, parity, indication for induction and initial bishop score (Table 1). 
Table 1: Demographic profile.

\begin{tabular}{|c|c|c|c|}
\hline Variable & $\begin{array}{l}\text { Sublingual } \\
\text { Misoprostol }\end{array}$ & $\begin{array}{l}\text { Vaginal } \\
\text { Misoprostol }\end{array}$ & $\begin{array}{l}\text { P } \\
\text { value }\end{array}$ \\
\hline \multicolumn{4}{|l|}{ Maternal age } \\
\hline$\leq 20$ & $05(10 \%)$ & $06(12 \%)$ & 1.0 \\
\hline $21-25$ & $30(60 \%)$ & $33(66 \%)$ & 0.68 \\
\hline $26-30$ & $14(28 \%)$ & $09(18 \%)$ & 0.34 \\
\hline$>30$ & $01(2 \%)$ & $02(4 \%)$ & 1.0 \\
\hline \multicolumn{4}{|l|}{ Parity } \\
\hline Primipara & $29(58 \%)$ & $33(66 \%)$ & \multirow{2}{*}{0.54} \\
\hline Multipara & $21(42 \%)$ & $17(34 \%)$ & \\
\hline \multicolumn{4}{|c|}{ Indication for induction } \\
\hline Oligohydramnios & $12(24 \%)$ & $09(18 \%)$ & 0.62 \\
\hline Postdatism & $14(28 \%)$ & $12(24 \%)$ & 0.82 \\
\hline Pre-eclampsia & $12(24 \%)$ & $12(24 \%)$ & 1.0 \\
\hline PROM & $12(24 \%)$ & $17(34 \%)$ & 0.3 \\
\hline \multicolumn{4}{|c|}{ Initial bishop score } \\
\hline$<4$ & $22(44 \%)$ & $19(38 \%)$ & \multirow{3}{*}{0.68} \\
\hline $4-6$ & $28(56 \%)$ & $31(62 \%)$ & \\
\hline Mean & 3.7 & 3.9 & \\
\hline
\end{tabular}

In this study, a single dose was required for induction in $38 \%$ of the patients in sublingual group and $30 \%$ in the vaginal group. After 3 doses, labor was induced in all the 50 cases of the sublingual route whereas 6 subjects still had unfavorable cervix in the vaginal route of misoprostol administration (Table 2).

Augmentation with oxytocin was required lesser for the sublingual group (26\%) as compared to the vaginal group (36\%). The mean time required for induction was 5 hours 55 minutes in the sublingual group and 7 hours 10 minutes in the vaginal group.

The sublingual group was associated with an increased incidence of induction within 4 hours; $19 / 50$ for sublingual vs $14 / 50$ for vaginal group. A sub-group analysis revealed that the sublingual misoprostol group was associated with a higher success in inducing labor in 12 hours: 50/50 for sublingual vs 44/50 for vaginal group (Table 2). In this study, 33 women delivered vaginally within 12 hours of induction in the sublingual and 25 in the vaginal groups. Also, 43 women in the sublingual group and 41 women in the vaginal group delivered vaginally within 24 hours.

Table 2: Outcome of induction.

\begin{tabular}{|c|c|c|c|}
\hline Variable & Sublingual Misoprostol & Vaginal Misoprostol & Statistics \\
\hline \multicolumn{4}{|c|}{ Doses required for induction } \\
\hline 1 & $19(38 \%)$ & $15(30 \%)$ & \multirow{3}{*}{$\begin{array}{l}\mathrm{P}=0.40 \mathrm{RR}-1.27,95 \% \mathrm{CI}-0.73 \text { to } \\
2.19\end{array}$} \\
\hline 2 & $24(48 \%)$ & $17(34 \%)$ & \\
\hline 3 & $07(14 \%)$ & $12(24 \%)$ & \\
\hline 4 & 0 & $04(8 \%)$ & \multirow{2}{*}{$\begin{array}{l}\mathrm{P}=0.02 * \mathrm{RR}-1.13,95 \% \mathrm{CI}-1.02 \text { to } \\
1.26\end{array}$} \\
\hline 5 & 0 & $02(4 \%)$ & \\
\hline $\begin{array}{l}\text { Augmentation with } \\
\text { oxytocin }\end{array}$ & $13(26 \%)$ & $18(36 \%)$ & $\begin{array}{l}\mathrm{P}=0.28 \mathrm{RR}-0.72,95 \% \mathrm{CI}-0.39 \text { to } \\
1.31\end{array}$ \\
\hline \multicolumn{4}{|c|}{ Time required for induction } \\
\hline$\leq 4$ & $19(38 \%)$ & $14(28 \%)$ & \multirow{4}{*}{$\begin{array}{l}\mathrm{P}=0.29 \mathrm{RR}-1.36,95 \% \mathrm{CI}-0.76 \text { to } \\
2.39\end{array}$} \\
\hline $4-8$ & $22(44 \%)$ & $18(36 \%)$ & \\
\hline $8-12$ & $9(18 \%)$ & $12(24 \%)$ & \\
\hline$\geq 12$ & 0 & $04(8 \%)$ & \\
\hline \multicolumn{4}{|l|}{ Mean duration } \\
\hline Initiation to induction time & 5 hours $55 \mathrm{~min}$ & 7 hours $10 \mathrm{~min}$ & $\mathrm{P}=0.14$ \\
\hline Induction delivery interval & 9 hours 10 mins & 11 hours 5 mins & $\mathrm{P}=0.19$ \\
\hline Duration of first stage & 8 hours 30 mins & 10 hours 30 mins & $\mathrm{P}=0.15$ \\
\hline
\end{tabular}

The mean interval from the start of induction to vaginal delivery was 9 hours 10 minutes in the sublingual misoprostol group and 11 hours 5 minutes in the vaginal misoprostol group (Table 2).

The mean duration of 1 st stage was 8 hours 30 minutes in the sublingual group and 10 hours 30 minutes vaginal group. But the difference was statistically not significant (Table 2).
Success rate was $100 \%$ in the sublingual group and $96 \%$ in the vaginal group. $84 \%$ cases in sublingual group and $82 \%$ cases in vaginal group delivered by normal vaginal delivery. There was $1(2 \%)$ instrumental delivery in sublingual group and the indication was fetal distress (Table 3). The cesarean section rate was $14 \%$ in the sublingual group and $18 \%$ in vaginal group. The indication of LSCS in sublingual group was fetal distress in all the cases (Table 3). 
Table 3: Labour outcome.

\begin{tabular}{|llll|}
\hline Variable & Sublingual Misoprostol & Vaginal Misoprostol & Statistics \\
\hline Failure of induction & $0(0 \%)$ & $2(4 \%)$ & $\mathrm{P}=0.30$ RR-0.2, 95\% CI- 0.0098 to 4.06 \\
\hline Mode of delivery & & & \\
\hline Normal delivery & 42 & 41 & $\mathrm{P}=0.59$ \\
\hline Instrumental & 1 & 0 & RR-0.77, $95 \%$ CI- 0.31 to 1.93 \\
\hline LSCS & $7(14 \%)$ & $9(18 \%)$ & \\
\hline Indication of LSCS & & & $\mathrm{P}=0.52$ \\
\hline Fetal distress & 7 & 4 & $\mathrm{P}=0.24$ \\
\hline Non progression & 0 & 3 & $\mathrm{P}=0.47$ \\
\hline Failure of induction & 0 & 2 & \\
\hline
\end{tabular}

Table 4: Side effects and complications.

\begin{tabular}{|llll|}
\hline Variable & Sublingual misoprostol & Vaginal misoprostol & Statistics \\
\hline Nausea and Vomiting & $3(6 \%)$ & $2(4 \%)$ & $\mathrm{P}=0.73$ RR- 1.25, CI- 0.36 to 4.38 \\
\hline NRFHR $*$ & $11(22 \%)$ & $8(16 \%)$ & $\mathrm{P}=0.45$ RR-1.37, CI-0.60 to 3.13 \\
\hline Meconium staining of liquor & $14(28 \%)$ & $11(22 \%)$ & $\mathrm{P}=0.49$ RR-1.27, CI- 0.64 to 2.53 \\
\hline Tachysystole & $5(10 \%)$ & $2(4 \%)$ & $\begin{array}{l}\mathrm{P}=0.26 \text { RR-2.5, 95\% CI-0.51 to } \\
12.29\end{array}$ \\
\hline Hypertonus & 0 & 0 & \\
\hline Hyperstimulation syndrome & $2(4 \%)$ & $1(2 \%)$ & \\
\hline
\end{tabular}

Nausea and vomiting was observed in 5 women in the sublingual group and 4 in the vaginal group. There were 11 cases of non-reactive fetal heart rate (NRFHR) in the sublingual group and 8 cases in the vaginal group. The incidence of meconium stained liquor was more in sublingual misoprostol group 14/50 vs 11/50 (Table 4). Hyperstimulation syndrome occurred in 2 cases in sublingual group as compared to a single case in vaginal group. In all the cases, intrauterine resuscitation was carried out and fetal heart rate pattern improved after which all subjects delivered vaginally (Table 4).

\section{DISCUSSION}

In this study, no significant difference observed in indication for induction between both the groups. In sublingual group, Postdatism was the most common indication for induction whereas in vaginal group it was PROM. The mean initial Bishop's score was 3.7 in sublingual group and 3.9 in the vaginal group thus, no significant difference was observed $(\mathrm{P}=0.68)$. Initial Bishop's score in sublingual group was $<4$ in $44 \%$ and $38 \%$ in vaginal group (Table 1 ).

In this study, more subjects went into labor after a single dose of sublingual misoprostol as compared to vaginal route. However, the difference was not statistically significant $(\mathrm{P}=0.40)$. A quicker onset of labor with sublingual route is probably explained by the pharmacokinetic studies that have shown that the peak plasma concentration and bioavailability after a single dose of misoprostol are higher after sublingual administration than those after vaginal administration. ${ }^{1-3}$ This may be explained by the absence of a first-pass effect by the liver after sublingual administration. The good blood supply under the tongue and the relatively neutral $\mathrm{pH}$ in the buccal cavity may also be contributing factors. The sublingual route was more efficacious in inducing labor after 12 hours of induction ( 3 doses) and the difference was statistically significant $(\mathrm{P}=0.02)$. In a study by Bartusevicus et al, single dose was required for induction in $50 \%$ of the patients in sublingual group and $27 \%$ in the vaginal group (RR-1.9, 95\% CI-1.2-2.9). ${ }^{7}$

The need for augmentation with oxytocin was lesser for the sublingual group (26\%) as compared to the vaginal group $(36 \%)$ (Table 2). But the difference was statistically not significant $(\mathrm{P}=0.28)$. In the study by Feitosa et al $29 \%$ in the study by Bartusevicus et al $49 \%$ in both the groups required augmentation with oxytocin. ${ }^{3,7}$ The mean time required for induction was 5 hours 55 minutes in the sublingual group and 7 hours 10 minutes in the vaginal group. Thus, the mean time required for induction was shorter in the sublingual group but the difference was statistically not significant $(\mathrm{P}=0.14)$ (Table 2).

As clearance of the drug is likely to be rapid irrespective of the route of administration, the prolonged activity of the vaginal and sublingual routes is presumably due to continued absorption over a long period. It would therefore be subject to the retention of the tablet in the respective site over a long time. A sub-group analysis revealed that sublingual misoprostol group was 
associated with a higher success in inducing labor in 12 hours; the difference was significant $(\mathrm{P}=0.02)$. The sublingual group was associated with an increased incidence of induction within 4 hours but the difference was not statistically significant $(\mathrm{P}=0.29)$ (Table 2).

When the occurrence of delivery within 24 hours was compared, sublingual misoprostol fared marginally better. Also, more subjects delivered within 12 hours in sublingual group but the difference was not statistically significant. In a similar study by Bartusevicus et al, in the sublingual group, 58 women $(83 \%)$, and in the vaginal group, 53 women $(76 \%)$, delivered vaginally within 24 hours. ${ }^{7}$ The number of women delivered vaginally within 12 hours of induction in the sublingual group were 13 (19\%) and vaginal $9(13 \%)$ groups. In this study, mean time required for induction to delivery $(\mathrm{P}=0.19)$ and mean duration of first stage $(\mathrm{P}=0.15)$ was shorter in the sublingual group than the vaginal group (Table 2). But the differences were statistically not significant. In the study by Bartusevicus et al, the interval from the start of induction to vaginal delivery was significantly shorter in the sublingual misoprostol. ${ }^{7}$

In the study, success rate of induction was $100 \%$ in sublingual group and $96 \%$ in vaginal group (Table 3). However, the difference was not statistically significant. $(\mathrm{P}=0.30)$ In this study, $84 \%$ delivered by normal vaginal delivery in sublingual group and $82 \%$ in vaginal group. The cesarean section rate was $14 \%$ in the sublingual group and $18 \%$ in vaginal group. The difference was not significant $(\mathrm{P}=0.59)$ (Table 3). In the study by Bartusevicus et al $76 \%$ in sublingual group and $77 \%$ in the vaginal group delivered by normal vaginal delivery with LSCS rate $17 \%$ in sublingual group $20 \%$ in vaginal group. ${ }^{7}$

In present study, the indication of LSCS in sublingual group was fetal distress in all the cases. Where as in vaginal group, apart from fetal distress (8\%); in $6 \%$ cases, the indication of LSCS was non-progression of labor and in $4 \%$ it was failure of induction (Table 3). However, the difference was not significant for any indication. In the study by Bartusevicus et al the indications of LSCS was fetal distress in $10 \%$ of the sublingual group and $11 \%$ of the vaginal group and the incidence of LSCS for non-progression was $7 \%$ in both the groups. $^{7}$ In this study, no significant difference was found in the incidence of nausea and vomiting in both the groups. NRFHR was seen in more subjects in sublingual group than in vaginal group, but the difference was not statistically significant. In this study, the incidence of meconium staining of amniotic fluid, tachycardia and hyperstimulation was higher in sublingual group but the differences were not statistically significant (Table 4). It is hypothesized that sublingual route would mimic the efficacy of the vaginal route by having a similar pharmacokinetic profile, while having less hyperstimulation by avoiding the cervical effects. However, the findings of this study suggest that avoidance of a direct effect on the cervix did not reduce the risk of excessive uterine activity. The other complications like precipitate labor, cervical tear, rupture of uterus, PPH were not observed in our study. In this study, patient acceptance was not studied. However, other studies have shown that sublingual route is better accepted than the vaginal route. ${ }^{8}$

Reviews carried out to compare the effects of the different doses of misoprostol administered vaginally and sublingually have suggested that there are no statistically significant differences with respect to the efficacy of the different routes of administration. ${ }^{9}$ Though sublingual route is a less invasive route than vaginal; it should be remembered that vaginal administration results in a local effect on the cervix that foster the desired physiological effects in terms of cervical ripening. There is insufficient clinical experience of the sublingual route to conclude as to the usefulness and potential advantages or drawbacks of this route of administration. The results of a larger number of controlled clinical and epidemiological trials will be needed before it is possible to make any sound recommendations.

The limitations of this study were that the sample size was small and this was not a blinded study.

\section{CONCLUSION}

The sublingual route of administration of misoprostol is comparable in efficacy and safety to the vaginal route for induction.

\section{Funding: No funding sources \\ Conflict of interest: None declared \\ Ethical approval: Not required}

\section{REFERENCES}

1. Tang OS, Schweer H, Seyberth HW, Lee SW, Chung Ho P. Pharmacokinetics of different routes of administration of misoprostol. Hum. Reprod. 2002;17(2):332-6.

2. Tang OS, Gemzell-Danielsson K, Ho $\mathrm{P}$ C. Misoprostol: Pharmacokinetic profiles, effects on the uterus and side effects. Int J Gynaecol Obstet. 2007;99:160-7.

3. Aronsson A, Bygdeman M, Gemzell-Danielsson K. Effects of misoprostol on uterine contractility following different routes of administration. Hum Reprod. 2004;19(1):81-4.

4. Tang OS, Schweer H, Seyberth HW, Lee SW, Chung Ho P. Pharmacokinetics of repeated doses of misoprostol. Hum. Reprod. 2009;24(8):1862-9.

5. Bartusevicius A, Barcaite E, Nadisauskiene R. Oral, vaginal and sublingual misoprostol for induction of labor. Int J Gynaecol Obstet. 2005;91(1):2-9.

6. Feitosa FEL, Sampaio ZS, Alencar CA Jr, Amorim MMR, Passini R Jr. Sublingual versus vaginal 
misoprostol for induction of labour. Int J Gynaecol Obstet. 2006;94:91-5.

7. Bartusevicius A, Barcaite E, Krikstolaitis R, Gintautas V, Nadisauskiene R. Sublingual compared with vaginal misoprostol for labour induction at term: a randomised controlled trial. BJOG. 2006;113:1431-7.

8. Nassar AH, Awwad J, Khalil AM, Abu-Musa A, Mehio G, Usta IM. A randomized comparison of patient satisfaction with vaginal and sublingual misoprostol for induction of labour at term. BJOG. 2007;114:1215-21.

9. Muzonzini G, Hofmeyr GJ. Buccal or sublingual misoprostol for cervical ripening and induction of labour. Cochrane Database of Systematic Reviews. 2004;4.Art. No.: CD004221.

Cite this article as: Akare MD, Patel PK. A comparison of sublingual with vaginal administration of misoprostol for induction of labor at term. Int $\mathbf{J}$ Reprod Contracept Obstet Gynecol 2017;6:1398-403. 